\title{
Investigating the effects of beta-amyloid on hippocampal signalling in Alzheimer's disease
}

\author{
Julia M Warburton ${ }^{1 *}$, Daniel J Whitcomb ${ }^{2,3}$, Krasimira Tsaneva-Atanasova ${ }^{4}$, Kei Cho ${ }^{2,3}$ \\ From 24th Annual Computational Neuroscience Meeting: CNS*2015 \\ Prague, Czech Republic. 18-23 July 2015
}

\begin{abstract}
Alzheimer's disease (AD) is the leading form of dementia and is characterised clinically by cognitive decline and impairments to memory function. The protein amyloid- $\beta$ $(\mathrm{A} \beta)$ is thought to be a key mediator of this neurodegeneration [1]. Mounting evidence suggests that soluble $A \beta_{1-42}$ oligomers $\left(\mathrm{oA} \beta_{1-42}\right)$ cause the impairments observed in early $A D$, which includes synapse loss and synaptic dysfunction. For example, oA $\beta_{1-42}$ inhibits hippocampal long-term potentiation (LTP) [2], an important synaptic plasticity thought to underlie memory formation in the brain [3]. We have recently found that postsynaptic infusion of oA $\beta$ into hippocampal neurons causes an increase in synaptic conductance attributed to changes in the excitatory glutamate receptor profile at the synapse. The relevance of this to synaptic dysregulation and consequence on hippocampal signalling is yet to be determined.

Here we take a computational approach to investigate how oA $\beta_{1-42}$ dysregulates signalling within the hippocampus. We first develop a biophysical model of synaptic potentiation in a CA1 neuron, based on a simple kinetic synapse model and Hodgkin-Huxley formalism [4], which successfully reproduces the conductance increase observed in single-cell patch clamp experiments following oA $\beta$ application. We then extend this to investigate how this affects signalling across a small network of CA1 neurons, analysing the importance of connectivity strength and number of affected neurons to the overall dynamics of the system. Recent studies have suggested that there are two distinct types of CA1 neurons with different electrophysiological properties [5]. Therefore we also consider the impact a heterogeneous population would have on signalling across the network.
\end{abstract}

\section{Acknowledgements}

This work was supported by funding from the EPSRC and Wellcome TrustMRC Neurodegenerative Disease Initiative Grant.

\section{Authors' details}

${ }^{1}$ Bristol Centre for Complexity Sciences, University of Bristol, Bristol, BS8 1TR UK. ${ }^{2}$ Henry Wellcome Laboratories for Integrative Neuroscience and Endocrinology (HW-LINE), University of Bristol, Bristol, BS1 3NY, UK. ${ }^{3}$ Centre for Synaptic Plasticity, University of Bristol, Bristol, BS1 3NY, UK. ${ }^{4}$ Department of Mathematics, University of Exeter, Exeter, EX4 4QF, UK.

Published: 18 December 2015

\section{References}

1. Hardy J, Selkoe DJ: The amyloid hypothesis of Alzheimer's disease: progress and problems on the road to therapeutics. Science 2002, 297(5580):353-356

2. Walsh D, Klyubin I, Fadeeva JV, Cullen WK, Anwyl R, Wolfe MS, Rowan MJ, Selkoe DJ: Naturally secreted oligomers of amyloid $\beta$ protein potently inhibit hippocampal long-term potentiation in vivo. Nature 2002, 416(6880):535-539.

3. Bliss TV, Collingridge GL: A synaptic model of memory: long-term potentiation in the hippocampus. Nature 1993, 361(6407):31-39.

4. Destexhe A, Mainen ZF, Sejnowski TJ: An efficient method for computing synaptic conductances based on a kinetic model of receptor binding. Neural Comput 1994, 6(1):14-18

5. Mizuseki K, Diba K, Pastalkova E, Buzáski G: Hippocampal CA1 pyramidal cells form functionally distinct sublayers. Nature Neurosci 2011, 14:1174-1181.

doi:10.1186/1471-2202-16-S1-P34

Cite this article as: Warburton et al:: Investigating the effects of betaamyloid on hippocampal signalling in Alzheimer's disease. $B M C$ Neuroscience 2015 16(Suppl 1):P34.

\footnotetext{
* Correspondence: julia.warburton@bristol.ac.uk

${ }^{1}$ Bristol Centre for Complexity Sciences, University of Bristol, Bristol, BS8 1TR, UK

Full list of author information is available at the end of the article
} 